\title{
Prostatectomía radical abierta, laparoscópica y robótica: ¿en busca de un nuevo gold standard?
}

\author{
Martínez-Salamanca JI*, Allona Almagro A**. \\ *Department of Urology. Weill Medical College at Cornell University. New York, USA \\ **Servicios de Urología. Hospital Universitario Ramón y Cajal. Facultad de Medicina. Universidad de Alcalá de \\ Henares. Hospital Ruber Internacional. Madrid.
}

Actas Urol Esp. 2007;31(4):316-327

\section{RESUMEN}

PROSTATECTOMÍA RADICAL ABIERTA, LAPAROSCÓPICA Y ROBÓTICA: ¿EN BUSCA DE UN NUEVO GOLD STANDARD?

Introducción: En bien conocido que la prostatectomía radical (PR) es una opción excelente como tratamiento del cáncer de próstata localizado, desde el punto de vista de control oncológico. Desde hace décadas los esfuerzos de la comunidad urológica en este campo, se centran en intentar mejorar los resultados funcionales (morbilidad urinaria y sexual) tras este procedimiento. Desde el inicio de este siglo, conviven tres abordajes (abierto, robótico y laparoscópico) que hasta nuestros días pugnan por intentar alcanzar y demostrar los mejores resultados. El objetivo de esta revisión ha sido hacer una puesta al día lo más exhaustiva, rigurosa y objetiva posible, sobre los resultados oncológicos y funcionales que consiguen las tres técnicas (abordajes) de PR.

Material y métodos: Hemos centrado la comparativa en cuatro apartados: resultados perioperatorios, resultados oncológicos, resultados funcionales y costes económicos. Para ellos se realizó unas búsqueda sistemática en los siguientes registros: PubMed, OVID, EMBASE y Cochrane Library, con los siguiente términos: PR Retropúbica, PR Abierta, PR Laparoscópica, PR Robótica, Función Sexual, Incontinencia Urinaria, Calidad de Vida, Costes Económicos.

Se seleccionaron un total de 73 referencias, a criterio de los autores, que fueron analizadas individualmente.

Resultados: La mortalidad relacionada con el procedimiento es extremadamente baja con cualquiera de las técnicas, con poco dolor postoperatorio y mínimos requerimientos analgésicos. Los resultados oncológicos, medidos en términos de márgenes quirúrgicos son comparables: PR Abierta (14-20\%), PR Laparoscópica (7,4-21,9\%) y PR Robótica (5,7-17,3\%). En relación a resultados funcionales (función sexual y continencia urinaria) es dificil establecer comparaciones debidos a la multitud de sesgos existentes (estudios no randomizados, diferentes métodos y escalas de medida, diferentes definiciones, etc).En los estudios uni-institucionales, los resultados parecen ser equivalentes.

Conclusiones: Las series de PR Laparoscópica y Robótica aún carecen de resultados maduros en términos de control bioquímico y resultados funcional a largo plazo. Parece que con estos abordajes la pérdida sanguínea y la necesidad de transfusión es menor que en cirugía abierta. La tecnología robótica aporta ventajas muy interesantes, que podrían tener un papel en homogeneizar los resultados del abordaje mínimamente invasivo, pero que aguardan validación a medio y largo plazo.

Palabras clave: Adenocarcinoma de próstata. Prostatectomía radical. Cirugía abierta. Laparoscopia robótica

\section{ABSTRACT}

RADICAL PROSTATECTOMY: OPEN, LAPAROSCOPIC AND ROBOTIC. LOOKING FOR A NEW GOLD STANDARD?

Introduction: It is well known that radical prostatectomy (RP) is an excellent option in localized prostatic cancer especially from oncological control point of view. The efforts, during last decades, of the urological community in this field have been addressed in trying to improve functional outcomes (urinary and sexual morbidity) after the procedure. From the beginning of this century, three managements (open, robotic and laparoscopic) have been coexisting trying to get and prove the best results. The objective of this review has been to make the most exhaustive, rigorous and objective updating with the functional and oncological outcomes from the three (RP) techniques.

Material and methods: We have centered the comparison in four sections: perioperative, oncological outcomes, functional results and economic costs. With this purpose a systematic search was made in the following registers: PubMed, OVID, EMBASE and Cochrane Library, with the following terms: Retropubic RP, open RP, laparoscopic RP, robotic RP, Sexual function, urinary incontinence, quality of life, economic costs.

At author's criteria, a total of 73 references were selected, that were individually analyzed.

Results: Whatever the technique is, the mortality related to the procedure is extremely low, with little postoperative pain and minimum analgesic requirements. The oncological results are similar, measured in surgical margin terms: Open RP (14-20\%), Laparoscopic RP (7.4-21.9\%) and robotic RP (5.7-17.3\%). Concerning functional results (sexual function and urinary continence), it is difficult to establish comparisons due to the multitude of existing byas (non randomized studies, different methods and measurement scales, different definitions, etc.) In the uni-insitutional studies, results seem to be equivalent.

Conclusions:Laparoscopic and robotic RP series are still pending of mature outcomes, related to long term biochemical control and functional results. It seems that with these managements, blood loss and transfusion needs are minor compared to open surgery. Robotic technology adds very interesting advantages that could have an important role in homogenize the minimally invasive management, but are still pending of validation at medium and long term.

Keywords: Prostate cance. Radical prostatectomy. Open laparoscopic. Robotic surgery. 
$\mathrm{E}^{\prime}$ cáncer de próstata representa una entidad oncológica única que durante más de un siglo ha fascinado a cirujanos que intentaban curar a sus pacientes mediante la "extirpación quirúrgica de la enfermedad”. Al parecer, la primera prostatectomía radical (PR), fue hecha en una Clínica de Zurich por Billroth en la década de 1860. La técnica original fue realizada por vía perineal. El éxito de este procedimiento en 40 pacientes inicialmente fue divulgado por Young hace más de 100 años y representó un hito en la cirugía oncológica. El abordaje retropúbico introducido por Millin en 1948 consiguió popularidad, especialmente por las ventajas que aporta un campo quirúrgico más amplio que proporciona flexibilidad para adaptar la cirugía a la anatomía individual del paciente, por un mejor control de la hemorragia y por la posibilidad de extirpar completamente el tumor en la mayoría de los pacientes. Sin embargo, la mejor comprensión de la anatomía pélvica a través de los trabajos de Walsh allanó el camino para estos procedimientos. En 1982, las modificaciones técnicas propuestas por Walsh y Donker en la preservación neurovascular en pacientes seleccionados, sin riesgo significativo sobre el control oncológico y preservando la función sexual y la continencia urinaria, supuso toda una revolución ${ }^{1}$. Todos estos principios representaban un reto a superar para cualquier técnica emergente.

En 1991, Schuessler et al describió la primera prostatectomía radical laparoscópica (PRL) ${ }^{2}$. Sin embargo, este impulso inicial se frenó debido a que 3 de los 9 pacientes tratados con PRL entre 1991 y 1995 experimentaron importantes complicaciones y estos autores concluyeron que aunque el procedimiento era factible técnicamente no ofrecía ninguna ventaja sobre la cirugía abierta en lo relativo a control tumoral, continencia, potencia sexual, duración de la hospitalización, convalecencia y resultados cosméticos ${ }^{2}$.

$\mathrm{El}$ arquetipo inicial de la prostatectomía radical se conmocionó ante la evidencia clínica generada en el Instituto Montsouris de Paris ${ }^{3-9}$ sobre la técnica descrita por Gaston en Burdeos ${ }^{3}$. En Montsouris la primera PRL fue realizada en Enero de 1998 y su posterior viabilidad técnica y los alentadores resultados de los pacientes tratados validaron su visión pionera estableciéndose el primer Servicio de Urología focalizado en la PRL. Estos meticulosos investigadores recogieron la información prequirúrgica, quirúrgica y perioperatoria y los resultados de los pacientes sometidos a PRL y describieron de forma decisiva los resultados de sus primeros 28, 40, 65 y 120 pacientes $^{4-9}$.

El Profesor Guillonneau enseñó su técnica por todo el mundo y diversos cirujanos se convirtieron en expertos en ella, validando la misma en centros en Francia, Alemania y Bélgica, extendiéndose posteriormente a Japón, Canadá y Estados Unidos. No obstante, se hizo evidente que el éxito del abordaje laparoscópico requería del desarrollo de importantes destrezas y habilidades de los cirujanos, problema que iba a quedar resuelto en un futuro con los adelantos de la tecnología en los laboratorios de robótica. Al igual que el PSA supuso una revolución en el avance en el diagnóstico precoz del cáncer prostático, la robótica revolucionó en Estados Unidos la cirugía radical prostática, consiguiendo combinar lo mejor del abordaje laparoscópico con las ventajas de la destreza técnica en cirugía abierta.

El grupo del Henry Ford Hospital (DetroitMichigan), liderado por el Dr. Menon, inició su andadura en esta técnica en el año 2001, quizás debido al poco éxito que consiguieron en la prostatectomía laparoscópica convencional, ya que inicialmente intentaron el desarrollo de un programa puro de PRL bajo la dirección y asesoramiento de los Dres. Guillonneau y Vallancien del Institut Mutualist Montsouris (París), pero después de un año, se demostró que el Dr. Menon, descrito coloquialmente por él, "fue incapaz" de realizar esta técnica con un nivel de excelencia. Dado que su equipo estaba razonablemente cómodo con el abordaje de la PRA descrita por Walsh-Lepor ${ }^{10,11}$ consideraron que podría ser útil la ayuda de la tecnología robótica para la laparoscopia. Menon había establecido contacto con el sistema da Vinci Medical Surgical System en Montsouris y recibió apoyo de los cirujanos franceses para intentar su aplicación en Detroit. Según los resultados preliminares obtenidos por el grupo de Abbou, Binder, Pasticier y cols. en 2001, el equipo de Henry Ford desarrolló un abordaje anatómico de la técnica de prostatectomía radical con ayuda robótica ${ }^{12,13}$. 
En la presente revisión los autores hemos intentado hacer una puesta al día lo más exhaustiva, rigurosa y objetiva posible, sobre los principales resultados oncológicos y funcionales que consiguen las tres técnicas de prostatectomía radical: abierta (realmente solo se incluye la vía retropúbica PRA), laparoscópica PRL y robótica PRR. Hemos dividido la comparativa en 4 apartados: resultados perioperatorios, resultados funcionales, resultados económicos y costes.

\section{RESULTADOS PERIOPERATORIOS}

Anestesia, postura, imposibilidades técnicas

Es indudable que en este aspecto la cirugía abierta muestra una clara ventaja, por poder ser realizada bajo anestesia regional, cosa imposible para las técnicas laparoscópicas. En estas últimas también hay que considerar la postura forzada del paciente en Trendelemburg (excepto en PRL extraperitoneal) durante un tiempo no despreciable, con las posibles consecuencias nocivas. Sin embargo, no hemos encontrado datos que valoren posibles complicaciones en relación con la anestesia ni con la postura forzada, aunque de modo excepcional esta última podría impedir la realización de la técnica laparoscópica, ante la intolerancia por parte del paciente.

En casos igualmente excepcionales, las técnicas laparoscópicas no pueden realizarse en pacientes con múltiples cirugías y pegadura intra-abdominales, cosa que dificultaría pero no impediría el abordaje abierto retropúbico aconsejando claramente la vía perineal.

\section{Tiempo operatorio}

La duración de la intervención ha sido uno de los grandes caballos de batalla del abordaje mínimamente invasivo, tanto laparoscópico como robótico. Los tiempos iniciales de las series laparoscópicas eran tremendamente largos ${ }^{14-17}$, aunque fueron mejorando a medida que avanzaba la curva de aprendizaje ${ }^{18,19}$. Con la cirugía robótica está sucediendo algo parecido, y los trabajos demuestran que el tiempo quirúrgico se acorta de manera significativa y exponencial cuando aumenta el número de casos. Los tiempo quirúrgicos en el inicio de la curva de aprendizaje robótica, parecen algo inferiores (Tabla 1a) a los comunicados inicialmente por los grupos de laparoscopia y muy similares a los tiempo medios de prostatectomía abierta de las series recientes.

En resumen, en manos experimentadas, los tiempos tienden a igualarse con cualquier abordaje y, en cualquier caso, las diferencias no parecen ser suficientemente importantes como para impactar en la morbilidad peri-operatoria.

\section{Pérdida sanguínea}

La mayoría de las publicaciones muestran una menor pérdida sanguínea intraperatoria con abordajes laparoscópicos (con o sin robot). La presión intraabdominal facilitada por el neumoperitoneo, colapsa vasos sanguíneos, fundamentalmente venosos, que son una de las fuentes más importantes de hemorragia durante la prostatectomía. Asimismo, en las técnicas endoscópicas, la sección del complejo venoso dorsal se rea-

Tabla 1a

Datos postoperatorios: abierta, laparoscópica y robótica

\begin{tabular}{|c|c|c|c|c|c|c|c|}
\hline & Autor & Año & № casos & $\begin{array}{c}\text { Tiempo } \\
\text { quirúrgico }(\mathrm{m})\end{array}$ & $\begin{array}{c}\text { Pérdida } \\
\text { sanguínea (ml) }\end{array}$ & $\begin{array}{c}\text { Transfusión } \\
\text { (\%) }\end{array}$ & $\begin{array}{c}\text { Reconversión } \\
(\%)\end{array}$ \\
\hline \multirow[t]{3}{*}{ PRA } & Lepor $^{53}$ & 2003 & 1024 & 129 & 805 & 8,2 & $\mathrm{SD}$ \\
\hline & Salomon ${ }^{54}$ & 2002 & 145 & 197 & $\mathrm{SD}$ & 26,2 & $\mathrm{SD}$ \\
\hline & Lerner $^{55}$ & 1995 & 1000 & $\mathrm{SD}$ & $\mathrm{SD}$ & 12 & $\mathrm{SD}$ \\
\hline \multirow[t]{3}{*}{ PRL } & Guillonneau $^{6}$ & 2001 & 240 & 239 & 402 & 10 & 2,5 \\
\hline & Rassweiler ${ }^{46}$ & 2003 & 219 & 288 & 1100 & 30,1 & 4,2 \\
\hline & Abbou [ ] & 2000 & 137 & 285 & $\mathrm{SD}$ & 2,9 & $\mathrm{SD}$ \\
\hline \multirow[t]{6}{*}{ PRR } & Menon $^{31}$ & 2004 & 1100 & 160 & $50-250$ & $0 \%$ & $0 \%$ \\
\hline & $\mathrm{Hu}^{56}$ & 2006 & 322 & 186 & 250 & $1,6 \%$ & $0,6 \%$ \\
\hline & Farnham-Smith ${ }^{20}$ & 2006 & 176 & $\mathrm{SD}$ & 191 & $0,5 \%$ & $0 \%$ \\
\hline & Joseph $^{57}$ & 2006 & 325 & 130 & 196 & $1 \%$ & $0 \%$ \\
\hline & Van Applendorn- & & & & & & \\
\hline & Costello ${ }^{58}$ & 2006 & 150 & 191 & $\mathrm{SD}$ & $2,6 \%$ & $0 \%$ \\
\hline
\end{tabular}


liza al final de la intervención, una vez que la próstata esta prácticamente extirpada. Son varias las series que comunican pérdidas menores a 100 o $200 \mathrm{ml}^{12,18,20,21}$.

Farnham et al. han publicado recientemente una comparación prospectiva, entre PRA Y PRR en términos de pérdida sanguínea ${ }^{20}$. Sus resultados fueron que la media de hematocrito en el momento del alta fue de 38\% tras PRR y de 33\% tras PRA.

La diferencia final la marca la necesidad o no de transfusión de hemoderivados. Como se refleja en la Tabla 1a, las cifras de transfusión son sensiblemente superiores en las series de cirugia abierta.

De cualquier manera, realizar una comparación objetiva no es sencillo, ya que existe una gran diferencia de criterios entre los diferentes trabajos publicados. Por ejemplo, hay cirujanos que utilizan un nivel predeterminado de hemoglobina en sangre para trasfundir, independientemente del estado del paciente. Otros, por el contrario, esperan a que el paciente presente síntomas. Por tanto, existe un sesgo en la comparación, basado en la diferencia en la práctica clínica individual. No se han publicado datos que demuestren diferencias respecto a necesidad de reintervenciones causadas por hemorragia, aunque es de esperar que, de existir diferencias, sean a favor de las técnicas laparoscópicas.

\section{Dolor postoperatorio}

Algunos estudios comparativos muestran una diferencia clara a favor de los abordajes laparoscópicos en cuanto a menor dolor postoperatorio $^{12,22}$. Recientemente el grupo de Vanderbilt, ha publicado un trabajo hospitalario prospectivo, en el cual no se observan diferencias estadísticamente significativas entre ambos grupos, lo que probablemente se explique por los bajos índices de dolor que presentan los pacientes con ambas técnicas (PRA y PRR) ${ }^{23}$.

Las incisiones cutáneas son muy similares en la PRL y PRR y producen mínimo dolor postoperatorio. Por otro lado, la incisión que se realiza en la PRA (laparotomía media infraumbilical) causa poca morbilidad, al no seccionar vientres musculares. Por tanto, la teórica ventaja en la disminución del dolor postoperatorio no es tan clara como en otros escenarios (p.e. cirugía renal).

\section{Estancia hospitalaria}

En líneas generales la optimización de la técnica de prostatectomía ha reducido de manera determinante la estancia posquirúrgica en las últimas décadas. La mayoría de los grupos tanto robóticos como laparoscópicos tienen un ingreso, en la mayoría de los casos, de entre 24 horas y 48 horas $^{12,24}$. Los motivos que habitualmente alargan el ingreso son debidos al íleo paralítico, que provoca intolerancia digestiva.

Las series de PRA y PR Perineal señalan ingresos algo superiores, aunque son muy comunes en la actualidad programas con estancia reducida, en torno a 48-72 horas.

De cualquier manera, se debe ser cauto a la hora de interpretar los tiempos de ingreso, debiendo cada profesional adaptar esto a cada paciente, a cada entorno socio-sanitario y a su propio criterio profesional. En sistemas sanitarios con una presión excesiva en las cifras de estancia, las expectativas creadas en el paciente antes de la cirugía tienen una importancia capital en su propia decisión del alta hospitalaria.

\section{DIAS DE SONDAJE}

Las series clásicas de prostatectomía abierta recomendaban entre 2 y 3 semanas de sonda ${ }^{11}$. A medida que el refinamiento en la técnica ha permitido hacer una anastomosis de mejor calidad, los días de sondaje han ido disminuyendo, hasta 7-10 días. (Tabla 1b). En las series clásicas de PRL, se mantiene la sonda entre 5 y 7 días.

La mejor visualización que proporcionan las técnicas laparoscópicas y fundamentalmente la robótica (magnificación e imagen tridimensional), están permitiendo realizar, en manos experimentadas, una anastomosis uretro-vesical de alta calidad, lo que permitiría retirar la sonda en un tiempo minimo, aunque no se debe olvidar que existe un componente de edema postoperatorio, asociado intrínsecamente a la agresión quirúrgica y que necesita un tiempo mínimo para resolverse, lo que explicaría la alta tasa de retenciones urinarias, $11 \%$ o más, que se observan en algunos grupos laparoscópicos que han propuesto retirar la sonda precozmente (23 días) ${ }^{25}$. 
Tabla 1 b

Datos postoperatorios: abierta, laparoscópica y robótica

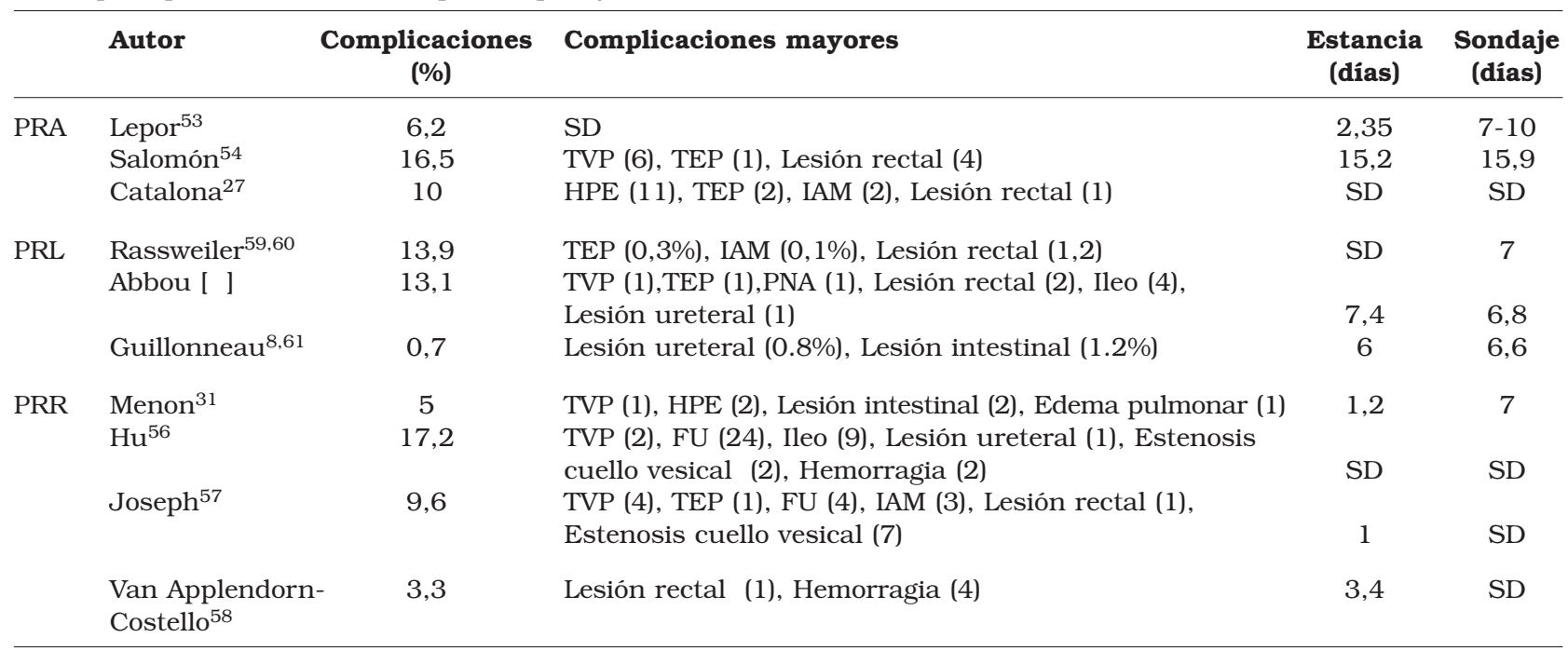

SD: Sin datos.

TVP: Trombosis venosa profunda, HPE: Hernia Puerto de entrada o incisional, TEP: Tromboembolismo pulmonar, PNA: Pielonefritis aguda, FU: Fístula urinaria, IAM: Infarto agudo de miocardio.

\section{RESULTADOS FUNCIONALES Continencia urinaria}

La técnica quirúrgica es un factor claramente implicado en la incontinencia de esfuerzo tras PR. La deficiencia intrínseca del esfínter es común en las primeras semanas, pero la gran mayoría de pacientes recupera a medio plazo la continencia $^{10,26-28}$.

El grupo de Menon publicó recientemente cómo la tecnología robótica permite una mejor visualización del ápex prostático y una disección más cuidadosa del mismo ${ }^{29}$. Se ha sugerido que la mínima agresión del ápex y, por tanto, de las fibras del esfinter estriado, puede tener un papel en la recuperación precoz de la continencia. Esta nueva observación abre un camino para intentar demostrar si la robótica puede aportar mejores resultados en disminuir el tiempo hasta la continencia ${ }^{30}$.

Los métodos de evaluación de la información de los trabajos publicados son muy diversos. Tanto la manera de recoger la información (cuestionarios, entrevista personal, contacto telefónico, etc.) como la definición de continencia asî como su gradación, hacen muy difícil establecer una comparación objetiva.

La realidad es que se han comunicado resultados excelentes en relación a la continencia uri- naria con cualquiera de las técnicas. Las mejores publicaciones, con idea de homogeneizar los datos, son las provenientes de estudios comparativos dentro de una misma institución.

Para hacer una idea al lector, exponemos en la Tablas comparativas, las cifras de continencia, definida como la completa continencia o la necesidad de usar como máximo un absorbente al día, que se obtienen a los 12 meses tras la cirugía por diversos autores, una vez superada la curva de aprendizaje (Tablas 2 y 3 ).

Como conclusión, podemos afirmar que no hay evidencia en la literatura para poder afirmar que un abordaje es claramente mejor en términos de obtención de continencia que otro.

\section{Función sexual}

Desde la descripción por Walsh y Donker ${ }^{1}$ de la anatomía de los nervios cavernosos y su aplicación a la prostatectomía radical, iniciando por tanto el concepto de preservación neurovascular (PNV), han sido muchos los avances que se han realizado en este aspecto funcional.

Es indiscutible que los principios de la disección de los haces neurovasculares deben ser los mismos, independientemente de la técnica o vía quirúrgica empleada. Es aún incierto el papel que puede jugar la nueva tecnología, en cuanto a 
Tabla 2

\begin{tabular}{|c|c|c|c|c|c|c|c|c|c|c|}
\hline & Autor & Año & No & & TNM (\%) & & Tasa global & & MQP (\%) & \\
\hline & & & casos & pT2 & pT3a & pT3b & MQP (\%) & pT2 & pT3a & pT3b \\
\hline PRA & Catalona $^{62}$ & 1998 & 1778 & $\mathrm{SD}$ & & & 20,9 & $\mathrm{SD}$ & & \\
\hline & Huland $^{63}$ & 2001 & 789 & $\mathrm{SD}$ & & & $\mathrm{SD}$ & 14,9 & & \\
\hline & Salomon $^{54}$ & 2002 & 145 & 62,1 & 17,2 & 20,6 & $\mathrm{SD}$ & 18,8 & & \\
\hline & Lepor $^{53}$ & 2003 & 1024 & $\mathrm{SD}$ & & & 20,5 & $\mathrm{SD}$ & & \\
\hline PRL & Abbou [ ] & 2000 & 137 & 75.9 & 13.8 & 10,2 & 28,4 & 21,9 & & \\
\hline & Guillonneau $^{5}$ & 2003 & 1000 & $\mathrm{SD}$ & & & $\mathrm{SD}$ & 15,5 & & \\
\hline & Rassweilwer ${ }^{64}$ & 2005 & 1078 & 59,2 & 21,4 & 17,6 & $\mathrm{SD}$ & 7,4 & & \\
\hline PRR & Patel $^{44}$ & 2005 & 200 & 78 & 14 & 8 & 10,5 & 5,7 & 26,2 & 33 \\
\hline & Menon 65 & 2005 & 35 & 98 & & & 2 & $\mathrm{SD}$ & $\mathrm{SD}$ & $\mathrm{SD}$ \\
\hline & Chien $^{66}$ & 2005 & 56 & 82 & & & 11 & $\mathrm{SD}$ & $\mathrm{SD}$ & $\mathrm{SD}$ \\
\hline & $\mathrm{Hu}^{56}$ & 2006 & 322 & 24,8 & 0,3 & 0 & $\mathrm{SD}$ & $\mathrm{SD}$ & $\mathrm{SD}$ & $\mathrm{SD}$ \\
\hline & Joseph $^{57}$ & 2006 & 325 & 81 & 14 & 5 & 13 & 9,9 & 37,1 & 27,3 \\
\hline & $\begin{array}{l}\text { Van Applendorn- } \\
\text { Costello }\end{array}$ & 2006 & 150 & $\mathrm{SD}$ & $\mathrm{SD}$ & $\mathrm{SD}$ & 17.3 & $\mathrm{SD}$ & $\mathrm{SD}$ & $\mathrm{SD}$ \\
\hline
\end{tabular}

SD: Sin datos.

Márgenes quirúrgicos positivos: abierta, laparoscópica y robótica.

Tabla 3

Continencia urinaria: abierta, laparoscópica y robótica

\begin{tabular}{|c|c|c|c|c|c|c|c|c|c|c|}
\hline & \multirow[t]{2}{*}{ Autor } & \multirow[t]{2}{*}{ Año } & \multirow{2}{*}{$\begin{array}{c}\text { No } \\
\text { casos }\end{array}$} & \multirow{2}{*}{$\begin{array}{c}\text { Definición de } \\
\text { continencia }\end{array}$} & \multirow{2}{*}{$\begin{array}{l}\text { Método } \\
\text { recogida } \\
\text { datos }\end{array}$} & \multicolumn{5}{|c|}{ Continencia (\%) } \\
\hline & & & & & & 3-meses & 6-meses & 12-meses & 18-meses & 24-meses \\
\hline \multirow[t]{3}{*}{ PRA } & Catalona $^{27}$ & 1999 & 1875 & No absorbente & Entrevista & & & & 92 & \\
\hline & Stanford ${ }^{67}$ & 2000 & 1291 & No absorbente & Cuestionario & & & & & 58,1 \\
\hline & Lepor $^{53}$ & 2003 & 1024 & 0-1 absorbente & Entrevista & & & 91 & & \\
\hline \multirow[t]{3}{*}{ PRL } & Abbou [ ] & 2000 & 137 & No absorbente & Cuestionario & 29 & 57 & 57 & & \\
\hline & $\operatorname{Eden}^{68}$ & 2002 & 100 & No absorbente & Entrevista & & & 90 & & \\
\hline & Rassweiler ${ }^{46}$ & 2003 & 219 & No absorbente & Entrevista & & & 90,3 & & \\
\hline \multirow[t]{4}{*}{ PRR } & Ahlering 47 & 2004 & 90 & No absorbente & Cuestionario & 81 & $\mathrm{SD}$ & $\mathrm{SD}$ & & \\
\hline & Costello 69 & 2005 & 122 & 0-1 absorbente & Cuestionario & 73 & 82 & $\mathrm{SD}$ & & \\
\hline & Patel $^{44}$ & 2005 & 200 & No absorbente & Cuestionario & 82 & 89 & 98 & & \\
\hline & Joseph ${ }^{57}$ & 2006 & 325 & No absorbente & Cuestionario & 93 & 96 & $\mathrm{SD}$ & & \\
\hline
\end{tabular}

SD: Sin datos

mejorar sustancialmente la calidad de visión de las estructuras anatómicas, permitiendo una disección casi microscópica, aunque varios autores que practican técnicas robóticas están comunicado cifras de preservación de la función sexual nunca publicadas antes con otras técnicas ${ }^{31-33}$.

Antes de describir algunas series publicadas, debemos tener en cuenta lo difícil que es comparar rigurosamente los resultados funcionales, sobre todo los tocantes a la función sexual. Así, podemos enumerar algunos aspectos controvertidos y variables en la metodología de los distintos estudios publicados:
- Evaluación preoperatoria de los pacientes con métodos diferentes (Cuestionarios (SHIM, IIEF), Entrevista, Cuestionarios propios de cada institución, etc.).

- Diferencias en el evaluador y en la forma de recogida de datos (Cuestionarios que rellena el paciente solo, otros que rellena el medico delante del paciente, entrevistas telefónicas, entrevista con la pareja, cuestionarios que se envian al paciente por correo postal, etc.).

- Diferencias en la definición de potencia pre y posquirúrgica. Existencia de diferentes puntos de corte sobre los mismos cuestionarios, Algunos 
estudios incluyen dentro del grupo con preservación de la función sexual tanto a pacientes sin tratamiento como a los que son potentes con Inhibidores de la Fosfodiesterasa tipo 5 (IPDE5) o con inyecciones intravenosas (IIC), y no se hacen diferencias según el medicamento o las dosis empleadas, etc.

En relación a los resultados tras PRA, Lepor et al. analizaron de manera prospectiva la función sexual (erección y coito) en una cohorte de varones menores de 50 anos. Encontraron que a los 12 meses el 83,5\% de ellos conseguía erecciones, sin tratamiento o usando IPDE5 o IIC, lo que ya conllevaba muchas dudas a la hora de evaluar estos resultados, sobre todo por incluir a pacientes respondedores a la terapia inyectable intracavernosa. Además, si el análisis se limitaba solo a la capacidad para realizar el coito, este porcentaje de éxito caía hasta el $64,5 \%{ }^{34}$. Por otro lado, los resultados asociados a PR Abierta suelen ser peores. Penson et al. encontraron que tan solo el $22 \%$ de los pacientes tenía erección suficiente como para realizar el coito tras 24 meses de seguimiento, llegando el porcentaje al $28 \%$ a los 60 meses, incluyendo igualmente a pacientes con $\mathrm{y} \sin$ medicación ${ }^{35}$.

Los resultados tras PRL fueron analizados en su serie por Stolzenburg et al., que realizan la técnica por vía extraperitoneal y con claro uso de electrocoagulación, encontrando un $12 \%$ de pacientes con erección suficiente para la penetración tras PNV unilateral y un $47 \%$ tras PNV bilateral, siempre tras 6 meses de seguimiento e incluyendo pacientes con tratamiento con IPDE5 ${ }^{36}$.

Su et al. publicaron en 2004 sus resultados tras PRL, describiendo que el $76 \%$ de sus pacientes sometidos e PRL con preservación neurovascular bilateral mantenía la erección suficiente para el coito tras 12 meses de seguimiento ${ }^{37}$. Rozet también comunicó sus resultados tras PRL, obteniendo un $43 \%$ de pacientes potentes tras PNV bilateral ${ }^{38}$.

Las series de PRR han comunicado en algunos casos (Tabla 4) unos resultados excelentes en cuanto a potencia sexual, usando nuevas técnicas,

Tabla 4

Función sexual: abierta, laparoscópica y robótica

\begin{tabular}{|c|c|c|c|c|c|c|c|c|c|c|}
\hline & Autor & Año & No & Técnica & Potencia & Recogida & & Poten & cia (\%) & \\
\hline & & & casos & & $\begin{array}{l}\text { (definida } \\
\text { como) }\end{array}$ & datos & 3 meses & 6 meses & 12 meses & 18 meses \\
\hline PRA & Catalona $^{27}$ & 1999 & 1875 & PRR & $\mathrm{CPP}$ & Entrevista & & & 66,5 & \\
\hline & Lepor $^{53}$ & 2003 & 1024 & PRR & $\mathrm{CPP}$ & Entrevista & & & 46,9 & \\
\hline & Stanford 67 & 2000 & 1291 & PRR & $\mathrm{CPP}$ & Cuestionario & & & & 56 \\
\hline & Stamey $^{70,71}$ & 1995 & 458 & PRR & $\mathrm{CPP}$ & Cuestionario & & & & 31,9 \\
\hline PRL & Turk $^{19}$ & 2001 & 44 & PRL & $\mathrm{CPP}$ & Entrevista & & & 59 & \\
\hline & Eden $^{68}$ & 2002 & 100 & PRL & $\mathrm{CPP}$ & Entrevista & & & 62 & \\
\hline & $\mathrm{Katz}^{72}$ & 2002 & 143 & PRL & $\mathrm{CPP}$ & Cuestionario & & & 53,8 & \\
\hline & Kaul-Menon ${ }^{33}$ & 2006 & 154 & $\begin{array}{c}\text { VIP } \\
\text { Velo de } \\
\text { Afrodita }\end{array}$ & $\mathrm{CPP}$ & SHIM & SD & $\mathrm{SD}$ & 96 & \\
\hline & Joseph $^{57}$ & 2006 & 325 & $\begin{array}{l}\text { Técnica } \\
\text { EX }\end{array}$ & IIEF $>21$ & IIEF-5 & 46 & $\mathrm{SD}$ & $\mathrm{SD}$ & \\
\hline & Chie $^{66}$ & 2005 & 56 & VIP & $\begin{array}{l}\text { Regreso } \\
\text { a la } \\
\text { situación } \\
\text { basal }\end{array}$ & UCLA-PCI & 54 & 66 & 69 & \\
\hline & Ahlering ${ }^{73}$ & 2005 & 36 & $\begin{array}{l}\text { Modified } \\
\text { VIP }\end{array}$ & $\mathrm{CPP}$ & IIEF-5 & 11 & SD & $\mathrm{SD}$ & \\
\hline & & 2005 & 23 & CFT & $\mathrm{CPP}$ & IIEF-5 & 47 & $\mathrm{SD}$ & $\mathrm{SD}$ & \\
\hline & Menon $^{74}$ & 2005 & 23 & VIP & $\mathrm{CPP}$ & IIEF-5 & SD & $\mathrm{SD}$ & 74 & \\
\hline & & 2005 & 35 & $\begin{array}{l}\text { Velo de } \\
\text { afrodita }\end{array}$ & $\mathrm{CPP}$ & IIEF-5 & $\mathrm{SD}$ & $\mathrm{SD}$ & 97 & \\
\hline
\end{tabular}

PRR: Prostatectomía Radical Retropúbica (Abierta); PRL: Prostatectomía Radical Laparoscópica; VIP: Vattikuti Institute Prostatectomy (Técnica Robótica); EX: Extraperitoneal; CFT: Cautery-Free Technique. CPP: Capacidad para penetración; IIEF: Índice Internacional de Función Eréctil; SHIM: Versión abreviada IIEF. UCLA-PCI: Universidad de California - Los Ángeles Prostate Cancer Index. SD: Sin datos 
como la de la Preservación del "Velo de Afrodita" del grupo de Detroit ${ }^{32}$ y la Técnica "Libre de Energia" de Tewari ${ }^{33}$.

Sin embargo, en un estudio comparativo de PRA y PRL, Namiki et al. llegan a la conclusión de que los pacientes intervenidos por vía abierta recuperan antes de la potencia sexual ${ }^{39}$.

En resumen, todavía es difícil llegar a conclusiones veraces debido a los grandes problemas metodológicos en la descripción de las series y a la ausencia de estudios prospectivos y randomizados, aunque es lógico albergar esperanzas de clara mejoría en los resultados funcionales sexuales conforme mejore la práctica de la robótica, ya que ésta ofrece unas claras ventajas en cuanto a visión que pueden redundar en una más exacta preservación de las bandeletas neurovasculares.

\section{RESULTADOS ONCOLÓGICOS}

Sin menospreciar los resultados funcionales, el primer objetivo en el tratamiento del cáncer de próstata localizado debe ser el control oncológico. Este control, lo podemos objetivar de dos maneras:

- Control a medio y largo plazo (Ausencia de recidiva bioquímica y/o progresión)

- Resultado inmediato tras la Prostatectomía (Primer valor de PSA y estado de los márgenes quirúrgicos de resección).

La PRA ha demostrado tras más de 15 años unos resultados excelentes, en cuanto a control oncológico global se refiere40-42, mientras que los datos a este respecto aún son limitados en cuanto a seguimiento en la PRL y más aún en la PRR.

En la serie de la Clínica Mayo de PRA, entre los años 1998 y 2003, las cifras porcentuales de pacientes libre de progresión bioquímica a los 3 y a los 5 años fueron del $99 \%$ y del $98 \%$ respectivamente $^{43}$. Esto puede por tanto considerarse insuperable.

Guillonneau et al., en su evaluación de resultados de una serie de 1000 pacientes sometidos a PRL, encontraron unos resultados de supervivencia libre de progresión bioquímica en relación al estadio patológico de $92 \%$ (pT2a), $88 \%$ (pT2b), 77 \% (pT3a) y $44 \%$ (pT3b) respectivamente ${ }^{5}$. Más recientemente, Rozet et al. publicaron en su serie unas cifras de supervivencia libre de recidiva bioquímica del $95 \%$ a 12 meses $^{38}$.
En cuanto a las series de PRR, aún debemos esperar más tiempo, pero existen resultados iniciales muy alentadores, como los comunicados por Patel et al. sobre un total de 200 pacientes con 9,7 meses de seguimiento (95\% de supervivencia libre de recidiva bioquímica), lo que apunta a que los logros podrían ser semejantes a los obtenidos con la técnica abierta ${ }^{44}$.

Al no existir seguimiento suficiente (sobre todo en las series de PRL y PRR) para evaluar con suficiente evidencia científica los resultados de supervivencia global y los de supervivencia libre de recidiva bioquímica, los resultados de los márgenes quirúrgicos tras la prostatectomía radical tienen un renovado interés como marcador de control oncológico.

Antes de revisar cualquier resultado, hay que considerar que, independientemente del tipo de abordaje, el número de márgenes positivos (MP) ha disminuido con el paso del tiempo debido a la migración de estadio en la era del PSA.

En todos los grupos la zona más frecuente de afectación de MP es la posterolateral.

Es bien conocido que la existencia de MP es un factor independiente que aumenta la probabilidad de progresión bioquímica y que impacta de manera negativa en la supervivencia ${ }^{40-42}$.

Las series clásicas de PR abierta retropúbica comunican unas cifras de MP discretamente superiores a las actuales con cualquiera de las tres técnicas (Tabla 2). Las series actuales de PRA $^{45-48}$ arrojan unas cifras de MP globales del 6,9 al 29\%. Para la PRL y PRR, las cifras de las series más recientes son muy parecidas (entre el 5,7 y el $37,1 \%$ ) (Tabla 2).

Brown et al. demuestran que no existen diferencias significativas relacionadas con la técnica en cuanto al porcentaje de MP entre la PRA y la PRL, ni entre la PRA y la PRR ${ }^{48}$.

En resumen, las tres técnicas parecen razonablemente comparables en su eficacia para erradicar la enfermedad local, pero es necesario un seguimiento mayor para poder compararlas en cuanto a supervivencia libre de progresión.

\section{COSTES ECONÓMICOS}

Es difícil hacer una aproximación a este tema ya que nuestro Sistema Nacional de Salud es muy diferente a otros en cuanto a costes, necesi- 
dades, etc, pero intentaremos dar una idea de la experiencia acontecida en otros países.

En los Estados Unidos se calcula que el coste del tratamiento global de los enfermos con cáncer de próstata en un año asciende a 1.300 millones de euros ${ }^{49,50}$. Es más, la PR representa el 50\% de ese desembolso.

Jayadevappa et al. ${ }^{51}$ calcularon el coste médico relacionado con los pacientes con nuevos diagnósticos de cáncer de próstata tratados con prostatectomía radical, incluyendo sus tratamientos adyuvantes. El coste anual era 3,8 veces mayor que el inducido en el grupo control que ellos habían establecido.

Trabajos más recientes, centrados en el análisis de costes económicos en la era de las terapias mínimamente invasivas para el cáncer de próstata, Link et al. ${ }^{49}$ demostraron que la PRL era 1,2 veces más costosa que la PRA. La diferencia de costes estaba asociada fundamentalmente al instrumental desechable. Si se utiliza material reesterizable durante el procedimiento, los pacientes se van de alta antes del segundo día y el tiempo operatorio es menos a 3,4 horas, los costes entre ambas técnicas se igualan.

En otro estudio comparativo de costes, Lotan et al. ${ }^{50}$, encontraron que la PRA ahorraba 387 euros con respecto a la PRL y 1.355 euros con respecto a la PRR. La gran diferencia observada con la robótica se debía a los costes de compra y mantenimiento del Robot.

En relación a los costes entre la PRA y la PRL, Guillonneau y Vallancien ${ }^{9}$ estimaron sobre un total de 100 pacientes, un ahorro medio de 1.237 dólares USA por caso. Esta diferencia se basaba en la reducción en la estancia hospitalaria en días, 6 para la PRL y 8 para la PRA respectivamente.

En resumen, en los tiempos actuales tanto la cirugía laparoscópica como la robótica tienen que intentar abaratar los costes del instrumental desechable (Trócares, Instrumental quirúrgico, dispositivos robóticos, mantenimiento, etc.) para poder competir económicamente de manera clara con los procedimientos abiertos ${ }^{75}$.

\section{CONCLUSIONES}

La prostatectomía radical en cualquiera de sus tres abordajes (abierta, laparoscópica o robó- tica) está absolutamente consolidada como tratamiento del cáncer de próstata localizado en enfermos con una expectativa de vida superior a 10 años.

Tras analizar las experiencias más importantes publicadas, se hace muy dificil poder establecer una comparación objetiva, ya que no existen series suficientemente significativas que enfrenten las tres técnicas de una manera prospectiva y randomizada.

Asimismo, la gran heterogeneidad en los instrumentos de medida (cuestionarios, entrevistas, etc.), en el seguimiento, en los criterios de selección de los enfermos y en la definición del status de los pacientes en cuanto a la función sexual y urinaria, hace prácticamente imposible establecer comparaciones reales.

Los resultados en cuanto a control oncológico, principal objetivo en el tratamiento de estos pacientes, son excelentes para la PRA y todos los datos señalan que también lo serán para las otras dos técnicas cuando haya pasado el tiempo suficiente para establecer una comparación equitativa.

Debemos también observar las tendencias y preferencias de los pacientes. Parece claro que los abordajes mínimamente invasivos se imponen. También se debe ser objetivo a la hora de alabar las ventajas de una u otra técnica, ya que cada cirujano debe basarse en sus propios resultados porque, sin duda, el factor cirujano es decisivo en los resultados funcionales, por encima de cualquier otro factor ${ }^{52}$, y ningún abordaje "per. $s e$ " condiciona unos resultados u otros.

La tecnología robótica, que ha desembarcado de manera decidida en este campo, aporta algunas innovaciones reales, entre las que destacan:

- La magnificación de los tejidos y su visualización tridimensional

- La mejora de la curva de aprendizaje del cirujano en comparación a la laparoscopia convencional

- La disección precisa de las estructuras anatómicas implicadas en los resultados funcionales (continencia y potencia)

Dejando a un lado el torbellino mediático que se genera alrededor de cualquier tecnología nueva y llamativa, la comunidad urológica tiene la responsabilidad de intentar demostrar si todos 
estos avances que la técnica pone a disposición del cirujano pueden ser de utilidad para mejorar la calidad de vida de nuestros pacientes intervenidos de PR, mejorando sus resultados funcionales.

El camino viene de la mano de evaluar, de manera prospectiva, multiinstitucional, con estudios bien diseñados y con instrumentos de medida veraces y reproducibles, las tres técnicas.

\section{REFERENCIAS}

1. Walsh PC, Donker PJ. Impotence following radical prostatectomy: insight into etiology and prevention. J Urol. 1982;128(3):492-497.

2. Schuessler WW, Schulam PG, Clayman RV, Kavoussi LR. Laparoscopic radical prostatectomy: initial short-term experience. Urology. 1997;50(6):854-857.

3. Gaston R. Comunicación personal. 1997.

4. Guillonneau B, Cathelineau X, Doublet JD. Vallancien G. Laparoscopic radical prostatectomy: the lessons learned. J Endourol. 2001;15(4):441-445.

5. Guillonneau B, el-Fettouh H, Baumert H, Cathelineau X, Doublet JD, Fromont G et al. Laparoscopic radical prostatectomy: oncological evaluation after 1.000 cases a Montsouris Institute. J Urol. 2003;169(4):1261-1266.

6. Guillonneau B, Rozet F, Barret E, Cathelineau X, Vallancien G. Laparoscopic radical prostatectomy: assessment after 240 procedures. Urol Clin North Am. 2001;28(1):189-202.

7. Guillonneau B, Rozet F, Cathelineau X, Lay F, Barret E, Doublet JD et al. Perioperative complications of laparoscopic radical prostatectomy: the Montsouris 3-year experience. J Urol. 2002;167(1):51-56.

8. Guillonneau B, Vallancien G. Laparoscopic radical prostatectomy: the Montsouris technique. J Urol. 2000;163(6): 1643-1649.

9. Guillonneau B, Vallancien G. Laparoscopic radical prostatectomy: the Montsouris experience. J Urol. 2000;163(2): 418-22.

10. Walsh PC. Anatomic radical prostatectomy: evolution of the surgical technique. J Urol. 1998;160(6 Pt 2):24182424.

11. Walsh PC. Anatomic radical retropubic prostatectomy in Campbell's Urology, P.C. Walsh, Retik B, Vaughan ED, Wein AJ, Editor. 1998, WB Saunders: Philadelphia:25652588.

12. Menon M, Tewari A, Baize B, Guillonneau B, Vallancien G. Prospective comparison of radical retropubic prostatectomy and robot-assisted anatomic prostatectomy: the Vattikuti Urology Institute experience. Urology. 2002;60 (5):864-868.

13. Menon M. Robotic radical retropubic prostatectomy. BJU Int. 2003;91(3): 175-176.

14. Kawabata G, Hara I, Hara S, Isotani S, Sakai Y, Wada Y et al. Laparoscopic radical prostatectomy: initial 17 case report. Nippon Hinyokika Gakkai Zasshi. 2001;92(7):647655.

15. Jacob F, Salomon L, Hoznek A, Bellot J, Antiphon P, Chopin DK et al. Laparoscopic radical prostatectomy: preliminary results. Eur Urol. 2000;37(5):615-620.

16. Hattori R, Ono Y, Gotho M, Yoshikawa Y, Hirabayashi S, Yamada S et al. Laparoscopic radical prostatectomy -initial 10 cases. Nippon Hinyokika Gakkai Zasshi. 2001;92(6): 603-608.
17. Habuchi T, Inuma M, Sato K, Matsuura S, Shimoda N, Tsuchiva N,et al. Early results in the initial 15 cases of laparoscopic radical prostatectomy in Akita University Medical Center. Hinyokika Kiyo. 2002;48(3):139-144.

18. Salomon L, Sebe P, De la Taille A, Vordos D, Hoznek A, Yiou R et al. Open versus laparoscopic radical prostatectomy: Part II. BJU Int. 2004;94(2):244-250.

19. Turk I, Deger S, Winkeimann B, Schonberger B, Loening SA. Laparoscopic radical prostatectomy. Technical aspects and experience with 125 cases. Eur Urol. 2001;40(1):4652.

20. Farnham SB, Webster TM, Herrel SD, Smith JA Jr. Intraoperative blood loss and transfusion requirements for robotic-assisted radical prostatectomy versus radical retropubic prostatectomy. Urology. 2006;67(2):360-363.

21. Smith JA Jr. Robotically assisted laparoscopic prostatectomy: an assessment of its contemporary role in the surgical management of localized prostate cancer. Am J Surg. 2004;188(4A Suppl):63S-67S.

22. Bhayani SB, Pavlovich CP, Strup SE, Dahi DM, Landman J, Fabrizio MD et al. Laparoscopic radical prostatectomy: a multi-institutional study of conversion to open surgery. Urology. 2004;63(1):99-102.

23. Webster TM, Herrell SD, Chang SS, Cookson MS, Baumgartner RG, Anderson LW et al. Robotic assisted laparoscopic radical prostatectomy versus retropubic radical prostatectomy: a prospective assessment of postoperative pain. J Urol. 2005;174(3):912-914.

24. Holzbeierlein JM, Smith JA. Radical prostatectomy and collaborative care pathways. Semin Urol Oncol. 2000; 18(1):60-65

25. Nadu A, Salomon L, Hoznek A, Olsson LE, Saint F, De la Taille A et al. Early removal of the catheter after laparoscopic radical prostatectomy. J Urol. 2001;166(5):16621664.

26. Smith JA Jr. Outcome after radical prostatectomy depends on surgical technique but not approach. Curr Urol Rep. 2002;3(3):179-181.

27. Catalona WJ, Carvalhal GF, Mager DE, Smith DS. Potency, continence and complication rates in 1.870 consecutive radical retropubic prostatectomies. J Urol. 1999;162(2): 433-438.

28. Fowler FJ Jr, Barry MJ, Lu-Yao G, Roman A, Wasson J, Wennberg JE. Patient-reported complications and followup treatment after radical prostatectomy. The National Medicare Experience: 1988-1990 (updated June 1993). Urology. 1993;42(6):622-629.

29. Menon M, Hemal AK, Tewari A, Shrivastava A, Bhandari A. The technique of apical dissection of prostate and urethrovesical anastomosis in robotic radical prostatectomy. BJU Int. 2004;93(6):715-719.

30. Takenaka A, Tewari A, Leung RA, Bigelow K, El-Tabey N, Murakami G et al. Preservation of the puboprostatic collar and puboperineoplasty for early recovery of urinary continence after robotic prostatectomy: anatomic basis and preliminary outcomes. Eur Urol. 2007;51:433-440.

31. Menon M, Hemal AK, VIP Team. Vattikuti Institute prostatectomy: a technique of robotic radical prostatectomy: experience in more than 1000 cases. J Endourol. 2004;18 (7):611-619.

32. Ahlering T, Eichel L, Chou D, Skarecky D. Feasibility study for robotic radical prostatectomy cautery-free neurovascular bundle preservation. Urology. 2005;65(5):994-997. 
33. Kaul S, Savera A, Badani K, Fumo M, Bhandari A, Menon M. Functional outcomes and oncological efficacy of Vattikuti Institute prostatectomy with Veil of Aphrodite nerve-sparing: an analysis of 154 consecutive patients. BJU Int. 2005;97(3):467-472.

34. Twiss C, Slova D, Lepor H. Outcomes for men younger than 50 years undergoing radical prostatectomy. Urology. 2005;66(1): 141-146.

35. Penson DF, McLerran D, Feng Z, Li L, Albertsen PC, Gilliland FD et al. 5-year urinary and sexual outcomes after radical prostatectomy: results from the prostate cancer outcomes study. J Urol. 2005;173(5):1701-1705.

36. Stolzenburg JU, Rabenalt R, DO M, Ho K, Dorschner W, Waldkirch E et al. Endoscopic extraperitoneal radical prostatectomy: oncological and functional results after 700 procedures. J Urol. 2005;174(4 Pt 1):1271-1275.

37. Su LM, Link RE, Bhayani SB, Sullivan W, Pavlovich CP Nerve-sparing laparoscopic radical prostatectomy: replicating the open surgical technique. Urology. 2004;64(1):123127.

38. Rozet F, Galiano M, Cathelineau X, Barret E, Cathala N, Vallancien G. Extraperitoneal laparoscopic radical prostatectomy: a prospective evaluation of 600 cases. J Urol. 2005;174(3):908-911.

39. Namiki S, Egawa S, Baba S, Terachi T, Usui Y, Terai A et al. Recovery of quality of life in year after laparoscopic or retropubic radical prostatectomy: a multi-institutional longitudinal study. Urology. 2005;65(3):517-523.

40. Humphreys MR, Gettman MT, Chow GK, Zincke H, Blute ML. Minimally invasive radical prostatectomy. Mayo Clin Proc. 2004;79(9):1169-1180.

41. Zincke H, Bergstralh EJ, Blute ML, Myers RP, Barret DM, Lieber MM et al. Radical prostatectomy for clinically localized prostate cancer: long-term results of 1.143 patients from a single institution. J Clin Oncol. 1994;12(11):22542263.

42. Ward JF, Slezak JM, Blute ML, Bergstralh EJ, Zincke H. Radical prostatectomy for clinically advanced (cT3) prostate cancer since the advent of prostate-specific antigen testing: 15-year outcome. BJU Int. 2005;95(6):751-756.

43. Gettman MT, Blute ML. Critical comparison of laparoscopic, robotic, and open radical prostatectomy: techniques, outcomes, and cost. Curr Urol Rep. 2006;7(3):193199.

44. Patel VR, Tully AS, Holmes R, Lindsay J. Robotic radical prostatectomy in the community setting-the learning curve and beyond: initial 200 cases. J Urol. 2005; 174(1): 269-272.

45. Anastasiadis AG, Salomon L, Katz R, Hoznek A, Chopin D, Abbou CC. Radical retropubic versus laparoscopic prostatectomy: a prospective comparison of functional outcome. Urology. 2003;62(2):292-297.

46. Rassweiler J, Seemann O, Schulze M, Teber D, Hatzinger M, Frede T. Laparoscopic versus open radical prostatectomy: a comparative study at a single institution. J Urol. 2003;169(5): 1689-1693.

47. Ahlering TE, Woo D, Eichel L, Lee DI, Edwards R, Skarecky DW. Robot-assisted versus open radical prostatectomy: a comparison of one surgeon's outcomes. Urology. 2004; 63(5):819-822.

48. Brown JA, Garlitz C, Gomella LG, Hubosky SG, Diamond SM, McGinnis D. Pathologic comparison of laparoscopic versus open radical retropubic prostatectomy specimens. Urology. 2003;62(3):481-486.
49. Link RE, Su LM, Bhayani SB, Pavlovich CP. Making ends meet: a cost comparison of laparoscopic and open radical retropubic prostatectomy. J Urol 2004;172(1):269-274.

50. Lotan Y, Cadeddu JA, Gettman MT. The new economics of radical prostatectomy: cost comparison of open, laparoscopic and robot assisted techniques. J Urol. 2004;172 (4Pt1):1431-1435.

51. Jayadevappa R, Bloom BS, Fomberstein SC, Wein AJ, Malkowicz SB. Health related quality of life and direct medical care cost in newly diagnosed younger men with prostate cancer. J Urol. 2005;174(3):1059-1064.

52. Bianco FJ Jr, Riedel ER, Begg CB, Catan MW, Scardino PT. Variations among high volume surgeons in the rate of complications after radical prostatectomy: further evidence that technique matters. J Urol. 2005;173(6):2099-2103.

53. Hsu EI, Hong EK, Lepor H. Influence of body weight and prostate volume on intraoperative, perioperative, and postoperative outcomes after radical retropubic prostatectomy. Urology. 2003;61(3):601-606.

54. Salomon L, Levrel O, de la Taille A, Anastasiadis AG, Saint F, Kaki S. Radical prostatectomy by the retropubic, perineal and laparoscopic approach: 12 years of experience in one center. Eur Urol. 2002;42(2):104-110.

55. Lerner SE, Blute ML, Lieber MM, Zincke H. Morbidity of contemporary radical retropubic prostatectomy for localized prostate cancer. Oncology (Williston Park) 1995;9(5): 379-382.

56. Hu JC, Nelson RA, Wilson TG. Perioperative complications of laparoscopic and robotic assisted laparoscopic radical prostatectomy. J Urol. 2006;175(2):541-546.

57. Joseph JV, Rosenbaum R, Madeb R, Erturk E, Patel HR. Robotic extraperitoneal radical prostatectomy: an alternative approach. J Urol. 2006;175(3 Pt 1):945-950.

58. Van Appledorn S, Bouchier-Hayes D, Agarwal D, Costello AJ. Robotic laparoscopic radical prostatectomy: setup and procedural techniques after 150 cases. Urology. 2006;67 (2):364-367.

59. Rassweiler J, Hruza M, Teber D, Su LM. Laparoscopic and robotic assisted radical prostatectomy - critical analysis of the results. Eur Urol. 2006;49(4):612-624.

60. Rassweiler J, Sentker L, Seemann O, Hatzinger M, Stock C, Frede T. Heilbronn laparoscopic radical prostatectomy. Technique and results after 100 cases. Eur Urol 2001; 40(1): 54-64.

61. Vallancien G, Cathelineau X, Baumert H, Doublet JD, Guillonneau B. Complications of transperitoneal laparoscopic surgery in urology: review of 1.311 procedures at a single center. J Urol. 2002;168(1):23-26.

62. Catalona WJ, Smith DS. Cancer recurrence and survival rates after anatomic radical retropubic prostatectomy for prostate cancer: intermediate-term results. J Urol 1998; 160(6 Pt 2): 2428-2434.

63. Huland H. Radical prostatectomy: options and issues. Eur Urol 2001; 39(Suppl 1): 3-9.

64. Rassweiler J, Schulze M, Teber D, Marrero R, Seemann O, Rumpelt $\mathrm{J}$ et al. Laparoscopic radical prostatectomy with the Heilbronn technique: oncological results in the first 500 patients. J Urol. 2005;173(3):761-764.

65. Menon M, Shrivastava A, Tewari A. Laparoscopic radical prostatectomy: conventional and robotic. Urology. 2005;66 (5 Suppl):101-104.

66. Chien GW, Mikhail AA, Orvieto MA. Modified clipless antegrade nerve preservation in robotic-assisted laparoscopic radical prostatectomy with validated sexual function evaluation. Urology. 2005;66(2):419-423. 
67. Stanford JL, Feng Z, Hamilton AS, Gililand FD, Stephenson RA, Eley JW et al. Urinary and sexual function after radical prostatectomy for clinically localized prostate cancer: the Prostate Cancer Outcomes Study. Jama. 2000;283(3):354-360.

68. Eden CG, Cahill D, Vass JA, Adams TH, Dauleh ML. Laparoscopic radical prostatectomy: the initial UK series. BJU Int. 2002;90(9):876-882.

69. Costello AJ, Haxhimolla H, Crowe H, Peters JS. Installation of telerobotic surgery and initial experience with telerobotic radical prostatectomy. BJU Int. 2005;96 (1):34-38.

70. Geary ES, Dendinger TE, Freiha FS, Stamey TA. Incontinence and vesical neck strictures following radical retropubic prostatectomy. Urology. 1995;45(6):1000-1006.

71. Geary ES, Dendinger TE, Freiha FS, Stamey TA. Nerve sparing radical prostatectomy: a different view. J Urol 1995;154(1):145-149.

72. Katz R, Salomon L, Hoznek A, De la Taille A, Vordos D, Cicco A et al. Patient reported sexual function following laparoscopic radical prostatectomy. J Urol. 2002;168(5): 2078-2082.
73. Ahlering TE, Eichel L, Skarecky D. Rapid communication: early potency outcomes with cautery-free neurovascular bundle preservation with robotic laparoscopic radical prostatectomy. J Endourol. 2005;19(6):715-718.

74. Menon M, Kaul S, Bhandari A, Shrivastava A, Tewari A, Hemal A. Potency following robotic radical prostatectomy: a questionnaire based analysis of outcomes after conventional nerve sparing and prostatic fascia sparing techniques. J Urol. 2005;174(6):2291-2296.

75. Martínez Salamanca JI, Allona Almagro, A. Número monográfico sobre cáncer de próstata. Enero 2007. (En Prensa).

Correspondencia autor: Dr. J.I. Martínez-Salamanca Clinical Research Fellow

Dept. of Urology. Brady Urological Institute Weill Medical College (Cornell University) New York, USA Tel.: 2127465874

E-mail autor: jum2013@med.cornell.edu Información artículo: Revisión

Trabajo recibido: enero 2007

Trabajo aceptado: febrero 2007 